\title{
Zur Appropriation sozio-technischer Systeme am Beispiel des Microblogging Systems Twitter
}

\author{
Nils Jeners, Peter Mambrey \\ Fraunhofer FIT, Schloss Birlinghoven, 53754 Sankt Augustin
}

\section{Einleitung und Fragestellung}

Technische Systeme werden gesellschaftlich produziert, das heißt durch Menschen auf Basis kultureller Erfahrungen, Werte und bestimmter Ziele. Das beginnt zuerst mit der Intention und ersten Realisierung vor dem kulturellen Hintergrund der Systementwickler und Auftraggeber. Der Kreis der Handelnden erweitert sich dann schnell durch die Aneignung und alltägliche Nutzung, also dem praktischen Einsatz durch Nutzer (Castells 2001). Dies ist kein linearer, kausaler und zielgesteuerter Prozess, sondern er ist vielfältigen - aus Sicht der Entwickler - externen Einflüssen unterlegen. Diese sind oft eher zufällig, nicht kalkulierbar, kreativ chaotisch und spiegeln das soziale Lernen (Williams 1997) in der Auseinandersetzung Mensch-Aufgabe-Werkzeug wider: neue Anwendungen, neue Bedarfe, neue Vorstellungen von Unterstützungen etc. Technikentwicklungen sind häufig innovativ und emergent, d.h. aus intentionalem Handeln entsteht etwas qualitativ Neues, das so nicht intendiert war. Wir bezeichnen diesen Vorgang als Appropriation, der Aneignung eines schon bestehenden Systems, dessen Nutzung und die Weiterentwicklung zu etwas Neuem. Das technikzentrierte Angebot des Microblogging Systems Twitter trifft in der alltäglichen Praxis auf die Aneignung und Nutzung durch eine Vielzahl von Anwendern. Wir wollen im folgenden Aufsatz aufzeigen, dass sich Twitter durch die Nutzungspraxis zu niedrigschwelligen kollaborativen Anwendungen hin öffnet und insbesondere durch den nebenläufigen Aufbau von Metawissen zukünftige Kollaborationen fördert.

Nimmt man die Ausgangsfrage von Twitter „What Are You Doing“ (WAYD) ernst, die seit 2006 von den Twitter-Entwicklern Jack Dorsey, Evan Williams und Biz Stone gestellt wird, müsste sich bei korrekter Antwort der Twitter-Anwender eine kollaborative Funktionsweise und Nutzung verschließen. Da geht es um persönliches Befinden und Empfinden. Die kollaborative Welt, die immer einen Bezug auf einen Anderen oder eine Gruppe darstellt, wird so nicht erreicht. Abweichend von der WAYD-Ausgangsfrage werden vielfältige Informationen in die Twitter-Welt gepostet, die mehr Inhalte transportieren, als die Information über das eigene augenblickliche Tun. Im Gegensatz zu den Nutzungserwartungen der 
Urheber findet so eine funktionale Erweiterung von Twitter statt. Dies erfolgt durch die Art und Weise der Nutzung durch die Anwender als Appropriation der Anwendung Twitter. Es steht zu erwarten, dass sich dieser funktionalen Erweiterung eine Anpassung der technischen Realisierung anschließt. So ließe sich dann wie bei anderen sozio-technischen Entwicklungen z.B. der SMS Funktion des Mobiltelefons (Livajic 2008) eine ko-evolutionäre, sozio-technische Innovation beobachten.

Das technikzentrierte Angebot einer Social Software trifft auf die Aneignung und Nutzung in der Praxis durch eine Vielzahl von Anwendern. Wir wollen im folgenden Aufsatz der Frage nachgehen, ob sich Twitter durch die Nutzungspraxis zu niedrigschwelligen kollaborativen Anwendungen hin öffnet und welche neuen Anforderungen dadurch an die Technikgestaltung entstehen.

\section{Einbettung in die Forschung}

Twitter ist ein Beispiel für eine technikzentrierte Innovation. Erst wurden von den Entwicklern Überlegungen zur technischen Funktionalität des Kommunikationsformates gemacht und dann im Versuch und Irrtum-Verfahren unterschiedliche Anwendungen getestet (Simon, Bernhardt 2008). Dadurch gelangte man zu der Anwendung, dass persönliche Befindlichkeiten oder aktuelle Wahrnehmungen per Microblogging veröffentlicht werden konnten. Seit den Arbeiten von Mackenzie und Waicman (1985) und Bijker und Law (1992) wird der Ansatz vertreten, dass aus technischen Artefakten soziale Produkte werden, die nicht technikimmanenten Gesetzen gehorchen, sondern durch eine Vielzahl von sozialen, kulturellen, ökonomischen und politischen Faktoren bestimmt werden. Technische Artefakte werden durch die Art und Weise ihres Zustandekommens konstruiert und dann im Zuge eines Appropriationsprozesses durch Aneignung und Nutzung in der alltäglichen Praxis durch eine Vielzahl von Anwendern weiterentwickelt. Hier treten oft Reibungen zwischen den technischen Artefakten und den Nutzungsvorstellungen der Anwender auf, die zu sozio-technischen Veränderungen führen. Das technische Artefakt wird modifiziert und in neue Nutzungsmodalitäten eingebettet. Diese Innovationen werden durch soziales Lernen in praktischer Auseinandersetzung mit der Technik hervorgerufen (Williams 1997).

Twitter ist ein sehr junges technisches Artefakt, es ist seit 2006 auf dem Markt. Wir folgen der Hypothese, dass sich aufgrund von sozialem Lernen die Art und Weise der praktischen Nutzung von Twitter geändert hat. Die ursprüngliche Intention wird überlagert von neuen Nutzungsformen. Wir folgen der Ansicht, dass zusätzlich zu der Idee des Broadcasting der Befindlichkeit oder Wahrnehmung einer Person ein Netcasting innerhalb von Gruppen hinzugekommen ist. Anders als Broadcasting, das die (sternförmige) Verbreitung einer Information darstellt, sehen wir Netcasting als interaktives, netzförmiges Komminkationsgeflecht. Dadurch wird direkte Kollaboration unterstützt und auch die Voraussetzungen für 
gelingende Kollaboration innerhalb der Gruppe gemeinsam geschaffen. Langdon Winner vertritt die These, dass wir Technikformate wählen, um die Art und Weise, wie wir leben, zu beeinflussen (Winner 1978). Informations- und Kommunikationstechniken sind unterspezifiziert und daher gestaltungsoffen. Diese „Elastizität“, so Castells macht es empfänglich für ganz unterschiedliche, teils gegenläufige Trends. Ob Gut oder Böse, es ist der Ausdruck unseres Selbst ausgedrückt in Kommunikationsformaten (Castells 2001). Durch die Art und Weise der Nutzung gestalten die Anwender in einer Wechselwirkung mit dem technischen Artefakt diese Technikformate mit. So geschieht es auch bei Twitter. Neben der anfänglichen Nutzung als Kommunikationsformat zur authentische Selbstdarstellung und vermarktung (Goffman 1959) sind weitere Nutzungen hinzu gekommen. Wir wollen die kollaborative Nutzung untersuchen.

\section{Vorgehensweise}

Um den Appropriationsprozess zu untersuchen, haben wir zwei Vorgehensweisen gewählt. Wir rekonstruierten aus der Literatur, aus dem Internet und aus anderen, persönlich zugänglichen Quellen Beispiele, die die Prozesse eindringlicher ausleuchten. Zusätzlich analysierten wir Updates innerhalb einer Zeitspanne. Zu einem Stichtag im Februar 2009 wurden 2750 öffentlich zugängliche und deutschsprachige Twitter-Updates aus dem Netz geladen und gespeichert. Sie bestanden aus drei Samples. Das erste betraf die Tweets derjenigen, die maximale Updates $(\mathrm{N}=27)$ aufwiesen. Das zweite wies eine maximale Zahl von Friends $(\mathrm{N}=55)$ auf, das dritte eine maximale Anzahl von Followers $(\mathrm{N}=60)$. Jeweils 20 Updates pro Twitter-Anwender wurden gespeichert. Nach der Kodierung erfolgte eine inhaltsanalytische Auswertung parallel durch drei Personen. Bei Bewertungsproblemen wurde im Zweifel nicht ausgewertet. Die inhaltliche Auswertung der Tweets erfolgte anhand von drei Kategorien: Selbstdarstellung: Im originalen Sinne der Twitter-Fragestellung: WAYD, dem wurden aber auch Witze; satirische Äußerungen; tagebuchartige Einträge etc. zugeordnet. Der persönliche Bezug war evident; Hinweise/Kommentierungen: In Bezugnahme auf einen Sachverhalt, der außerhalb der Person des Twitter-Anwenders war. Der außerpersönlicher Bezug war evident; Frage: Erbitten einer Auskunft durch andere. Explizites Feedback von der Außenwelt war evident.

Zusätzlich wurden Sonderzeichen in den Tweets analysiert wie die Zahl der direkten Adressierungen (@) der Hashtags (\#), der Links, der Re'Tweets (RT) und Fragen wie Zeitraum der Tweets, authentifizierbarer Anwender, Ort der „Location“, Anzahl der Favoriten etc. In diesem Aufsatz nutzen wir nur die empirisch gewonnen Erfahrungen, die sich auf eine kollaborative Nutzungserweiterung beziehen. 


\section{Ergebnisse}

Die Ausgangsfrage von Twitter, was man persönlich gerade mache, wurde nur noch von 38\% der Tweets beantwortet. Zu einem ähnlichen Ergebnis kommt Mischaud (2007). Hinweise und Kommentierungen erreichten fast 58\%, wobei in der internen Unterscheidung dreimal mehr Hinweise als Kommentare abgegeben wurden. Mit knapp 4\% war die Anzahl der Fragen, die sich an die Außenwelt richtete, sehr gering. Daraus kann man schließen, dass Twitter-Anwender eine andere Vorstellung von der Nutzung dieses Kommunikationsformates haben, als anfangs die Entwickler (Simon, Bernhardt 2008). Da 62\% aller Anwender unserer Erhebung die Ausgangsfrage negieren und etwas Anderes schreiben, wird Twitter von Anwendern grundsätzlich neu definiert. Statt dem Veröffentlichen von persönlichen Befindlichkeiten werden nun neue Funktionen von den Anwendern durch Nutzungspraxis neben die alte, intendierte Funktion gesetzt. Nutzungspraxis und intendierte Funktion entwickeln sich auseinander. Es bleibt abzuwarten, ob und wie von den technisch Verantwortlichen auf diese Entwicklung reagiert wird.

Die Idee, alle Twitter-Anwender an seinem Fühlen und Tun teilzuhaben „Guten Morgen, bin auf dem Weg zur Arbeit" wird ergänzt um adressatenspezifische Ansprache, um mit dem Update zwar alle Twitter-Anwender aber insbesondere eine ganz bestimmte Person anzusprechen (@name als direkte Anrede), also spezielle Aufmerksamkeit zu erregen.

Dies ist ein Schritt hin zur niedrigschwelligen Kooperation. In 28\% aller Tweets fanden sich adressatenspezifische Ansprachen, ein Indiz dafür, dass sich diese Twitter-Anwender von dem Ashton-Kutcher-Exhibitionismus entfernt haben und ihnen neue zusätzliche Funktionen wichtig sind. Es geht ihnen nicht nur um Selbstdarstellung, sondern dabei auch um die zielgerichtete Herstellung von Aufmerksamkeit. Dies ist ein Schritt in Richtung gedachte Kollaboration, man verbindet sich mit einer Aussage und wird so identifizierbar. Diese Selbstinszenierung kann die Glaubwürdigkeit erhöhen, da der Twitter-Anwender ja autonom über die 140 Zeichen verfügt und es von seiner Geschicklichkeit abhängt, ob die Rolle von den anderen Twitter-Anwendern geglaubt wird oder eben nicht (Goffman 1959, S. 19). Die textuelle Ausdruckskontrolle hat er jedenfalls.

$45 \%$ aller Tweets schlossen Links mit ein. Diese Verweiskultur hat einen deutlichen Vorteil: Man befreit sich von der Beschränkung auf 140 Zeichen und kann auf viel umfangreichere Informationen verweisen. Die Zeichen werden dann quasi nur als Werbebanner genutzt, der auf weitergehende Inhalte aufmerksam macht. Hier zeigt es sich, dass durch einfache softwaretechnische Vorkehrungen wie Links insbesondere auch die Nutzung von Kurz-URLs die Restriktionen umgangen werden können, ein kluger Weg der Circumvention bzw. Invention von neuen Features bei Beibehalten der bisherigen Beschränkungen. Es zeigt sich aber aufgrund der hohen Prozentzahl, dass fast der Hälfte der Twitter-Anwender der Zeichenvorrat von 140 Zeichen als nicht ausreichend erscheint, die für erforderlich gehaltenen Informationen zu versenden. Hier reibt sich die Designstrategie, ein mobiles, 
niedrigschwelliges Kommunikationsformat für kurze Mitteilungen einzusetzen an den Bedarfen und Gewohnheiten der Anwender. Es wird interessant werden zu sehen, ob diese Beschränkungen auch in Zukunft eingehalten werden oder durch neue textbasierte Konventionen wie z.B. das Verkürzen von Text durch Kürzel wie brb (be right back), thx (= thanks), n8(= night) oder durch neue technische Realisierungen wie z.B. TinyURL, der Umwandlung von langen Verweisen in kurze ersetzt werden.

In unseren Samples ist die Zahl der ReTweets (RT), der Wiederholung von Tweets anderer und das Veröffentlichen an den eigenen Leserkreis, sehr gering: Sie liegt bei $2 \%$. Das lässt darauf schließen, dass Twitter-Anwender sich nicht als Multiplikator von Meldungen verstehen, sondern eher eigene Inhalte anbieten. Da nicht geprüft werden kann, ob Meldungen anderer einfach nur paraphrasiert werden, kann man Twitter-Anwendern zumindest unterstellen, dass sie ihre Tweets für originell halten und hinter ihnen stehen.

Im Detail: Die drei Gruppen, die wir ausgewählt haben, unterscheiden sich deutlich voneinander. Die Anwender, die als Freunde abonniert wurden (max. Follower), gaben nur zu weniger als einem Drittel (30\%) über persönliches Befinden Auskunft. Dies unterschied sich deutlich von der Gruppe der „Freundeverfolger“ (max Friends) die zur Hälfte (50\%) sich selbst zum Gegenstand der Tweets machten.

Wie lässt sich dies interpretieren? Wer vielen Freunden folgt, erzählt viel von sich, wer viele Freunde hat, beschränkt sich eher auf Hinweise und Kommentare. Möglicherweise sind die Twitter-Anwender an außerpersönlichen Informationen stärker interessiert, als an persönlichen Informationen eines anderen Users. Dieses Ergebnis spricht gegen die Voyeurs- oder Selbstdarstellungshypothese. Bei Twitter finden Hinweise und Kommentare häufiger statt als persönliche Selbstdarstellung. In den Printmedien wird Twitter oft als Kommunikationsformat zur Verbreitung einer persönlichen Peepshow reduziert. Viele sogenannte Promis leben dies sicher auch aus aber es ist nicht die dominante Nutzung, die vorherrscht. Nach unseren Erhebungen scheint Twitter eher ein Medienformat zur Kommentierung von Ereignissen oder Dingen aus der individuellen Perspektive des Twitter-Anwenders zu sein. Es gibt mehr individuelle Verweise auf Außerpersönliches als die persönliche Darstellung. Dieses Ergebnis wird durch eine Auswertung der Verwendung von Hashtags und Links bestätigt. Diejenigen, die viele Freunde hatten, verwendeten zu über zwei Dritteln (71\%) Verweise (\# und Links), während in der Gruppe der Freundeverfolger dies nur die Hälfte (51\%) machten. Diese wandten sich auch seltener direkt an anderen Personen (durch Benutzung des @). Diejenigen, denen gefolgt wurde, nutzen häufiger (30\%) diese Funktion, die Folgenden nutzen sie weniger $(22 \%)$. Das Bild des selbstverliebten TwitterAnwenders, der Privates in die voyeuristische Twitter-Gemeinschaft hineinpostet, wird deutlich ergänzt um ganz unterschiedliche Nutzungen. Egozentrische Motive der Nutzung werden so ergänzt um altruistische Nutzungen. 


\section{Beispiele der Twitter Entwicklung}

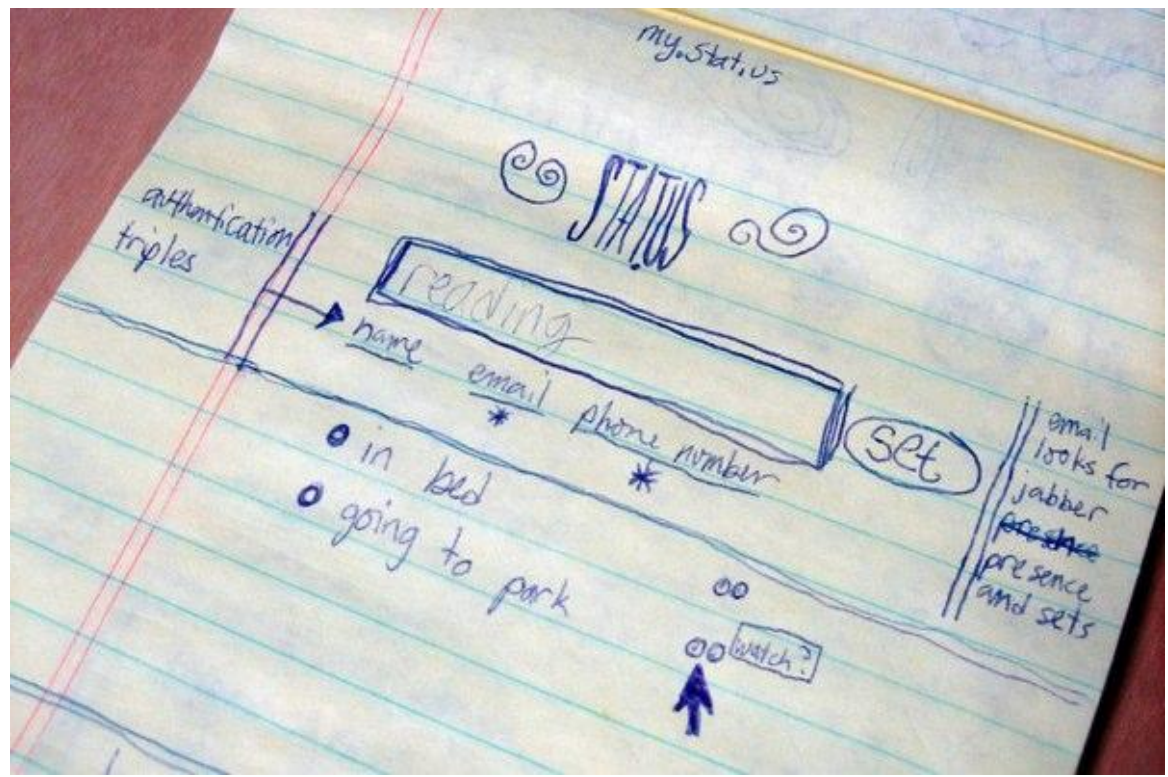

Abbildung 1: Papier-Prototyp von Twitter

Die ersten Prototypen von Twitter (Abbildung 1) zeichneten sich durch eine extrem limitierte Funktionalität aus. Die Beschränkung auf 140 Zeichen und der geringe Funktionsumfang machten die Benutzer erfinderisch. Mithilfe der beiden Programmierschnittstellen ${ }^{1}$ (REST-API und Search-API) bauten Nutzer die benötigen Funktionen um Twitter herum. Der größte Teil der Entwicklungen entstand tatsächlich durch die Twitter-Nutzer selbst. Durch das einfache Konzept und die API ist Twitter offen für Erweiterungen. Twitter absorbierte gute Ideen und integrierte sie in das eigene Konzept. Eine vollständige Sammlung aller Entwicklungen, die in und um Twitter entstanden sind, ist ausgeschlossen, da die Übersicht fehlt und die Entwicklungen zu dynamisch sind. Die Beschränkung auf ausgewählte Beispiele zeigt exemplarisch die Ausprägungen der verschiedenen Arten von Diensten, die neben Twitter existieren und/oder in Twitter integriert wurden.

Zu Beginn gab es in Twitter keine Suche. Der Datenstrom war ein Abbild des gegenwärtigen Moments und man sah nicht die Notwendigkeit in der Vergangenheit zu suchen. Summize ${ }^{2}$ bot genau diesen Dienst extern an. Es durchsuchte die Tweets und machte diese Ergebnisse Twitter-Nutzern verfügbar. Der Dienst wurde so beliebt, dass Twitter diesen im Juli 2008 kaufte und in das eigene Angebot integrierte.

\footnotetext{
1 http://apiwiki.twitter.com

${ }^{2}$ http://search.twitter.com
} 
Mit der Suche wurden weitere Funktionen notwendig. Schlicht durch die Masse der Tweets wurden diese trotz einer ausgeklügelten Suche schwerer zu bewältigen. Die Kategorisierung der Tweets durch Tags half hier weiter. Da Twitter diese Funktion nicht bereitstellte, fügten die Benutzer ein Rautenzeichen (engl. Hash) ein, um Tags zu kennzeichnen (z.B. \#MKWI für diese Konferenz). Hashtags ${ }^{3}$ durchsuchte die Tweets nach diesen Tags und baute einige Funktionen darauf auf. Durch Zählen der vorkommenden Tags ergeben sich sogenannte Hot Topics, die auf Twitter diskutiert werden. Diese Hashtags wurden mittlerweile ebenfalls in Twitter integriert, so dass alle Hashtags als Link dargestellt werden. Dieser Link führt zur Durchsuchung aller Tweets nach diesem Hashtag. Der Benutzer erhält die letzten Tweets, die dieses Hashtag enthalten, also z.B. alles zum Thema \#wahl. Zum Anlass aktueller Wahlen wurden die Hashtags selbst erweitert. Hashtags mit Parteinamen können mit + oder - ergänzt werden. Dadurch zeichnet sich ein politisches Stimmungsbild ${ }^{4} \mathrm{ab}$, das sicherlich nicht repräsentativ aber dennoch interessant ist.

Eine weitere Entwicklung, die auf dieser Suche nach Hashtags aufbaut sind Twitterwalls. Sie werden häufig bei größeren Ereignissen (z.B. Podiumsdiskussionen) eingesetzt, um Kommentare aus dem Publikum anzuzeigen. Ein vorher vereinbartes Hashtag wird genutzt, um Tweets auf dieser Twitter-Wand darzustellen. Die Tweets dienen einem Moderator als Rückkanal, so dass ein Dialog mit dem Publikum entstehen kann.

Twitter besitzt kein eigenes Gruppenkonzept. Die Nutzerkonten sind entweder öffentlich oder privat. Alle Follower können Tweets lesen. Eine Steuerung der Tweets ist so nur mit verschiedenen Nutzerkonten möglich (z.B. Arbeit, Sportverein, Familie). Dieser Mechanismus ist allerdings etwas umständlich und benötigt pro Nutzerkonto eine separate Emailadresse. GroupTweet ${ }^{5}$ versucht dieses Problem zu lösen und führt Gruppenkonten ein, die ähnlich wie Emailverteile funktionieren. Bei Twitter unterscheiden diese Konten sich nicht von herkömmlichen Benutzerkonten bieten aber eine Erweiterung, die auf dem Server von GroupTweet zur Verfügung gestellt wird. Alle Mitglieder folgen dem Gruppenkonto und umgekehrt. Direkte Nachrichten an das Gruppenkonto werden von dem Gruppenkonto automatisch erneut verschickt, sodass alle Follower diese Nachricht erhalten. Um den Absender kenntlich zu machen wird vor diese Nachricht ein@, gefolgt von dem Absender, eingefügt.

Das@ mit Benutzername ist ebenfalls eine Erfindung der Twitternutzer. Da Twitter kein gerichtetes Medium ist, brauchte man etwas, um Nutzer direkt anzusprechen, deren Tweets zu kommentieren oder Fragen zu beantworten. Twitter behandelt @ ähnlich wie \#. Es fügt einen Link ein, aber im Gegensatz zu \# wird keine Suche ausgeführt, sondern die Seite des jeweiligen Benutzers mit seinen letz-

\footnotetext{
3 http://hashtags.org

${ }^{4}$ http://www.wahlgetwitter.de und http://parteigezwitscher.de

5 http://grouptweet.com
} 
ten 20 Tweets wird angezeigt. Für Wiederholen (ReTweet) von Tweets, wird RT und dann@ mit Benutzernamen verwendet. Dies zeigt an, dass der Tweet nicht von dem Benutzer selbst verfasst, sondern nur weitergeleitet wurde, um es unter seinen Followern zu verteilen. Das RT ist eine Konvention unter den TwitterNutzern, die Twitter in seinen FAQ (Frequently Asked Questions) aufgenommen hat.

Die Beschränkung auf 140 Zeichen macht es schwierig Deep Links (Verknüpfungen in tiefere Ebenen einer Website) zu veröffentlichen, da Links selbst auch aus deutlich mehr als 140 Zeichen bestehen können. Kurz-URL-Dienste helfen genau bei diesem Problem. Sie kürzen die Links, indem sie einen kurzen Link auf ihre Seite (z.B. http://tr.im/mkwi10) auf die Eigentliche umleiten. Die Umwandlung in Kurz-URLs geschieht bei Twitter automatisch mit dem Dienst bit.ly ${ }^{6}$. Der Nutzer, der diesen Link getweetet hat, kann bei einigen Kurz-URL-Diensten eine Statistik seiner Links einsehen und so kontrollieren wie oft dieser Link aufgerufen wurde. Manche Kurz-URL-Dienste aggregieren diese Statistiken, um Links bzw. Themen herauszufiltern die in Twitter stark diskutiert werden. Ein Nachteil dieser Dienste ist, dass der Nutzer nicht auf den ersten Blick erkennen kann, wohin dieser Link führt. Diesem sicherheitskritischen Aspekt haben sich wiederum andere Dienste angenommen. LongURL ${ }^{7}$ wandelt die Kurz-URL wieder in die originale URL um.

Hashtags, und RT kamen von außen und fanden mehr oder weniger ihren Weg in Twitter und wurden integriert. Andere Entwicklungen koexistieren neben Twitter und nutzen nur die Daten, die Twitter zur Verfügung stellt. Diese TwitterMashups benutzen die Twitter-API, holen sich die Daten aus dem System und setzen sie neuartig wieder zusammen, oder kombinieren sie mit weiteren APIs. Twittervision ${ }^{8}$ stellt beispielsweise Tweets auf einer Landkarte dar. TweetStats ${ }^{9}$ und TwitterCounter ${ }^{10}$ zeigen eine Analyse (z.B. zeitlicher Verlauf der Follower) über einzelne Twitterkonten. TweetVolume ${ }^{11}$ analysiert die Häufigkeit bestimmter Schlüsselwörter in Twitter. Der Twitter-Browser ${ }^{12}$ stellt die Freunde eines Nutzers als Netzwerk dar, das navigierbar ist.

Neben den Diensten, die Daten aus Twitter herausholen und diese weiterverarbeiten, existieren auch Dienste, die ihre Daten automatisch in Twitter einfügen. Sogenannte Bots tweeten automatisch. Einfache Bots nehmen Datenquellen, die sie regelmäßig bei Twitter veröffentlichen, z.B. Wetterdaten einer bestimmten Region, Börsendaten von bestimmten Aktiengesellschaften oder Indices. Aufwändigere Bots interagieren mit anderen Benutzern. Ein Timer-Bot speichert direkte

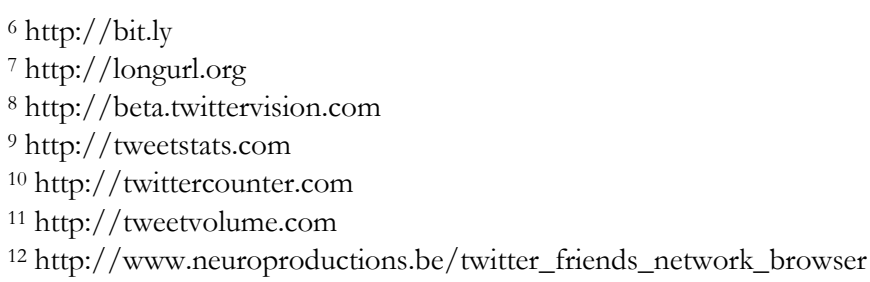


Nachrichten, die mit einer Uhrzeit beginnen und sendet die Nachricht um diese Uhrzeit wieder an den Sender zurück.

\section{Diskussion und Ausblick}

Die Ergebnisse der Untersuchung zeigen, dass Kollaboration durch Twitter technisch unterstützt wird: Es gibt die direkte Frage, die direkte Ansprache einer oder mehrerer Personen bzw. Organisationen, die direkte Aufforderung etwas gemeinsam zu tun und das gemeinsame Arbeiten an einem Produkt. So ist es im Zusammenhang mit den Wahlvorgängen im Iran weltweit zu politischen Protestaktionen durch Flash Mobs gekommen, die mittels Tweets organisiert wurden (z. B. http://bit.ly/S63q9). Versammlungsfreiheit ist ein hohes Gut in demokratischen Gesellschaften und muss in vordemokratischen Gesellschaften häufig erst politisch durchgesetzt werden. Twitter bietet einzelnen Bürgern die technische Möglichkeit, Versammlungen zu organisieren. Hierbei werden die Vorteile dieses niedrigschwelligen Angebots genutzt: Mobilität, Aktualität, geringer Ressourcenaufwand und direkte Awareness (Vergegenwärtigung). Sinnferne Aktionen (Flash Mobs) und niedrigschwellige Kommunikationsformate (Microblogging) können sich in ihrer Verbindung zu Organisationsmitteln in der politischen aber auch gesellschaftlichen Auseinandersetzung evolutionär hin verändern und eindrucksvoll genutzt werden, z.B. als Mittel beim Arbeitskampf Berlin 2007 (Landesarbeitsgericht Berlin Az.: Sa 967/08). Es finden also Funktionserweiterungen statt, die wir auf alltägliche Nutzungspraktiken und soziales Lernen zurückführen können. Aber zusätzlich zum Einsatz als Informations- und Organisationsmedium werden Voraussetzungen für eine gelingende zukünftige Kollaboration geschaffen:

Durch die Nutzung erfolgt ein nebenläufiger Aufbau von Metawissen, welches zukünftige Kollaboration erleichtert. Ganz im Sinne des partizipativen, peripheren Lernens (Lave, Wenger 1991) wird ohne konkretes Ziel durch Lesen von Tweets wahrgenommen, was anderen wichtig ist und wie sie es beurteilen. Hier findet ein Agenda Setting und Framing (Lakoff 2004) durch jeden einzelnen TwitterAnwender statt, welches der Leser der Tweets wahrnimmt und auf Basis seiner Erfahrungen interpretiert. Tweets sind damit Teil der virtuellen Außenwelt jedes einzelnen Twitter-Anwenders. Sie unterstützen ihn bei seiner interpersonellen Koorientierung innerhalb seiner Referenzgruppe (Chaffee 1975). Ob diese Informationen in seine Sicht der Außenwelt eingehen, ist eher wahrscheinlich, da er ja bewusst und freiwillig den Tweets folgt.

Ebenso wird wahrgenommen, wie Andere sich selbst zum Thema machen und darstellen. Dadurch entsteht die ,negotiated experience of self“ (Wenger 1998) die durch Reflexion der Darstellung Anderer oder durch direkte Teilnahme in einer virtuellen Gemeinschaft entsteht. Metawissen ist für die Kontrolle und Steuerung der eigenen Handlungen (Herrmann, Mambrey, Shire 2003, S. 41) nötig und fördert so das Gelingen von zukünftigen Kollaborationen. Es reduziert Unsicherheit 
und bietet eine Wissensbasis über Personen, die im Bedarfsfall abgerufen werden kann.

Die Selbstorientierung in einer selbst ausgewählten Gemeinschaft von Referenzpersonen beinhaltet aber auch Risiken. Da man bei Twitter diese Gemeinschaft völlig autonom zusammenstellen kann (durch das Folgen bzw. das Blocken), besteht die Gefahr, dass man sich einen eigenen Referenzrahmen schafft. Die autonome Selektion der eigenen Wissensbasis kann dann dazu führen, dass kritische Sichtweisen ausgeblendet werden. Da jeder sich seine eigene fragmentierte Twitter-Öffentlichkeit aufbauen kann, entfällt der Zwang zur Wahrnehmung anderer Positionen oder Interessen.

Die Flexibilität beim Einstieg und Ausstieg bei anderen Twitter-Anwendern oder Gruppen und die Unverbindlichkeit bei der Kollaboration, die daraus entsteht, macht deutlich, dass Twitter eine andere Form von "Community“ etabliert, als sie zu Beginn dieses Jahrhunderts z.B. von Preece (2000) beschrieben wurde. Es geht um volatile Netzbeziehungen, die kein Administrator pflegt, sondern der autonom handelnde Twitter-Anwender selbst. Diese Flexibilität kann man auch mit Beliebigkeit gleichsetzen, da keine Einigung auf einen Kompromiss oder eine Lösung zwingend ist. Eine zentrale Kontrolle und Steuerung ist deshalb nicht möglich, es sei denn über die Entwickler und Eigentümer selbst, die dadurch aber riskieren würden, dass auf andere Microblogging Systeme migriert würde, z.B. dem Open Source System laconi.ca.

Welche Gestaltungsanforderungen sich an die technische Weiterentwicklung stellen, hängt von der Art und Weise der Nutzung ab. Bisherige Gestaltungselemente haben sich bewährt: der niedrigschwellige Einsatz, der durch den geringen Ressourceneinsatz (Einarbeitungs- und Lernaufwand; Kosten; mobile Zugänglichkeit etc.) gefördert wird. Der Anspruch auf Aktualität, der suggeriert, man würde in Echtzeit handeln, die Unverbindlichkeit der Netzbeziehungen, die vom TwitterAnwender aufgenommen und wieder abgebrochen werden können, wobei die Datenspur weiter bestehen bleibt.

Die Kürze der Tweets garantiert den schnellen Überblick über hunderte eingehender Tweets, ohne daraus einen zeitaufwändigen Beruf zu machen. Dem Einzelnen fehlt aber die Transparenz über das gesamte Netzgeschehen und er gibt seine Tweets irreversibel in die Netzöffentlichkeit ab.

Wenn wir unsere Erfahrungen mit dem Wissen über Appropriation und Technikentwicklung vergleichen, bestätigen sich viele Erkenntnisse:

Es lässt sich keine Zukunft der sozio-technischen Anwendung prognostizieren, zu viele Faktoren gerade auf der Nutzerseite bestimmen über Annahme und Nutzung des Angebots. Lineare Pfade diffundieren zu einem dynamischen Wechselspiel aus technology-push, social-call, technology-push etc.;

Die Offenheit der Systementwickler für Neuerungen scheint den dynamischen Appropriationsprozess zu unterstützen und zu einem auf die Nutzerbedarfe angepassten Produkt zu machen. Dabei wechselt die Initiative von Entwicklern zu Nutzern aber auch wieder zurück; 
Das augenscheinliche Fehlen eines Geschäftsmodells und die Kostenfreiheit suggeriert das Vorhandensein eines öffentlichen, gemeinsamen Guts, das frei genutzt und gestaltet werden kann. Dies scheint die Kreativität bei der Aneignung zu unterstützen;

Das Entwicklungsprinzip der „Leichtgewichtigkeit“ trifft auf die Vorstellungen und Nutzungsgewohnheiten der Nutzer und fördert diese. Geringe Voraussetzungen, geringe Bindungen sowie ein nutzerautonome Vernetzung suggerieren dem Nutzer, dass er Herr des sozio-technischen Systems ist.

\section{Literatur}

Bijker, W.; Law, J. (1992) (eds.) Shaping Technology/Building Society: Studies in Socio-technical Change. MIT Press Cambridge/MA and London.

Castells, M. (2001) The Internet Galaxy. Reflections on the Internet, Business, and Society. Oxford Univrsity Press New York, p. 6.

Chaffee, Steven (1975) The Diffusion of Political Information. In: Chaffee, Steven (ed.): Political Communication. Issues and Strategies for Research.Sage Publications Beverly Hills, CA, pp. 95.

Goffman, Erving (1959) The Presentation of Self in Every Day Life. Doubleday \& Company, Inc. New York.

Herrmann, Thomas; Mambrey, Peter; Shire, Karen (2003) (Hrsg.): Wissensgenese, Wissensteilung und Wissensorganisation in der Arbeitspraxis. Westdeutscher Verlag Wiesbaden.

Honeycutt, C.; Herring, S. C. (2009) Beyond Microblogging: Conversation and Collaboration via Twitter. In: Proceedings of the Forty-Second Hawai'i International Conference on Systems Sciences (HICSS-42), Los Alamitos, CA: IEEE-Press.

Lakoff, George (2004) Dont't Think of an Elephant - Know Your Values and Frame the Debate. Chelsea Green Publishers.

Lave, Jean,; Wenger, Etienne (1991) Situated Learning: Legitimate Peripheral Participation. Cambridge University Press Cambridge UK.

Livajic, Denis (2008) Mobiltelefon im öffentlichen Raum - Nutzungen und Praktiken im Umgang mit dem Mobiltelefon. Diplomarbeit Universität Duisburg-Essen 2008.

MacKenzie, D. ; Waicman, J. (1985) (eds.): The Social Shaping of Technology: How the Refrigerator Got Its Hum. Open University Press Milton Keynes. 
Mischaud, Edward (2007) Twitter: Expressions of the whole self. An investigation into user appropriation of a web-based communications platform. London.

MSc. Thesis.

http://www.lse.ac.uk/collections/media@lse/mediaWorkingPapers/MScDiss ertationSeries/Mischaud_final.pdf retrieved June 26, 2009.

Preece, Jenny (2000) Online Communities: Designing Usability, Supporting Sociability. John Wiley and Sons, Chichester.

Simon, Nicole; Bernhardt, Nikolaus (2008) Twitter - Mit 140 Zeichen zum Web 2.0. Open Source Press München.

Time (2009) June 15 pp. 29.

Wenger, Etienne (1998) Communities of Practice. Learning, Meaning, and Identity. Cambridge University Press Cambridge UK.

Winner, Langdon (1978) Autonomous Technology: Technics-out-of-Control as a Theme in Political Thought. The MIT Press Cambridge MA.

Williams, Robin (1997) The Social Shaping of Information and Communications Technology. In: Kubicek, H.; Dutton, W. H.; Williams, R. (eds.): The Social Shaping of Information Superhighways. Campus Verlag Frankfurt/New York, pp. 299-338. 